\title{
Development of Gear Design Software Package
}

\author{
Shubham Khilari" ${ }^{*}$, Harsh Kakashaniya ${ }^{\#, ~ A s h i s h ~ K a r a n j e ", ~ V a r u n ~ J a d h a v \# ~ a n d ~ S a n d e e p ~ T h o r a t ~}{ }^{\#}$ \\ \#Mechanical Engineering Department, MIT College of Engineering, Savitribai Phule Pune University, Pune, India \\ Accepted 02 March 2016, Available online 15 March 2016, Special Issue-4 (March 2016)
}

\begin{abstract}
This report presents an overview of the Gear Design software package developed by us. This package was developed as an attempt to dive into interdisciplinary pool of engineering, Often during designing gears for gearboxes or other transmission systems, the engineer faces the problem of extracting information from standard data-books, manually calculating the forces and torques and then solving for module. This process is highly inefficient and time-consuming and does not guarantee optimized design or does not allow the engineer to view the variations in parameters at a single glance. Thus in order to make the engineer's job easy this package has been developed using fundamental programming practices and open source language, Python. The package features an ergonomic graphical user interface(GUI). It automatically generates 3D CAD model of the gear pair and also auto-generates a logically complete industrial grade report.
\end{abstract}

Keywords: Gear Design, CAD/CAM, Python, programming, software package, optimization, graphical user interface, spur gears.

\section{Introduction}

Gears are an integral part of many important mechanical systems. They are mainly used to transmit power, reduce speed, increase torque and relieve load from the main motor shaft. Their design can greatly influence durability, reliability and maintainability of a system, as well as its overall weight, As a result, it is becoming increasingly important to develop the best gear design for a given situation, as system design criteria become increasingly stringent. This paper introduces the reader to a novel way of designing efficient spur gear pair without indulging in complex and time-consuming calculations.

The backend programming part is entirely coded in Python programming language. We chose Python as it is easy to learn and is highly efficient for creating prototypes. The graphical user interface is also written in Python which helped us to easily connect the backend program to the frontend code. Also Python has many open source modules which helped us to efficiently manipulate data and process and present it in a professional way.

The gear design process uses both the bending strength and wear principle to calculate optimum module. Firstly the condition for failure is checked by the initial script. If the bending strength is less than wear strength, bending failure criteria is used otherwise failure due to wear criteria is used.
Accordingly, primary module is calculated by considering velocity factor method. This module is then used in the Buckingham method to check for safety. Module is incremented by a specific value till the safety condition is met.

After module is calculated, this module is used calculate all the geometrical and dynamic properties of the gear namely, addendum, dedendum, face width, Maximum gear tooth force, etc.

These values are then exported to report generation program which generates a complete industrial grade report which provides all the necessary information about the gear pair.

Simultaneously, the calculated module and number of teeth are exported to CAD generation program which generates a script to automatically create a 3D CAD model of the gear pair.

\section{Method and Output}

The method used in designing the package is logical and can be presented in the form of flowchart.

Input parameters:

1. Power (in kW)

2. Gear Ratio

3. Input RPM

4. Number of teeth on pinion (default 18)

5. Gear and pinion strength( $\mathrm{MPa})$

6. Tooth Type

7. Service Factor $\left(K_{a}\right)$

8. Load Concentration factor $\left(\mathrm{K}_{\mathrm{b}}\right)$ 
9. Young's Modulus of gear and pinion

10. Face width factor

11. Manufacturing Grade

12. Factor of Safety(FOS)

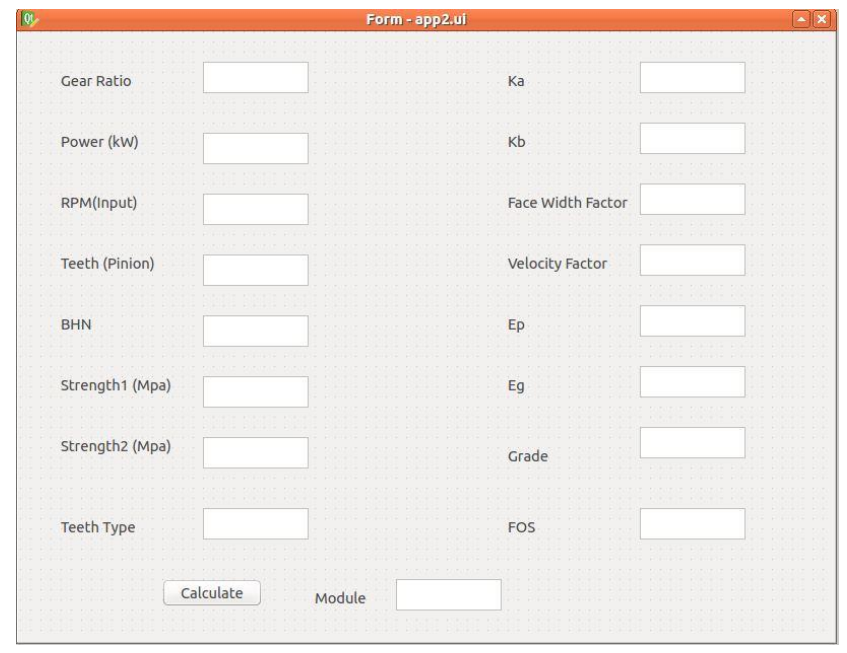

Fig. 1 Graphical User Interface of Prototype

When the input values are given to the user interface the "Calculate" button executes the complete backend program which can be illustrated by the following flowchart.

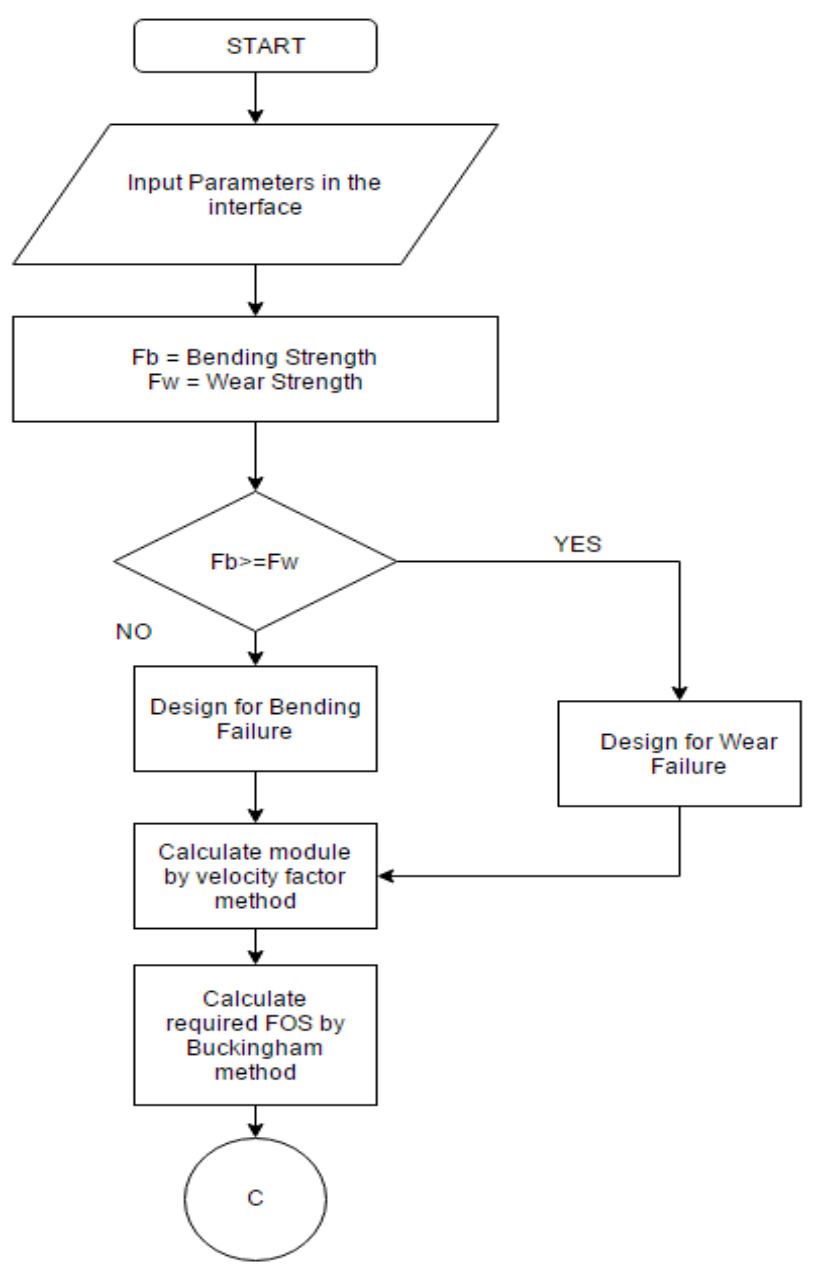

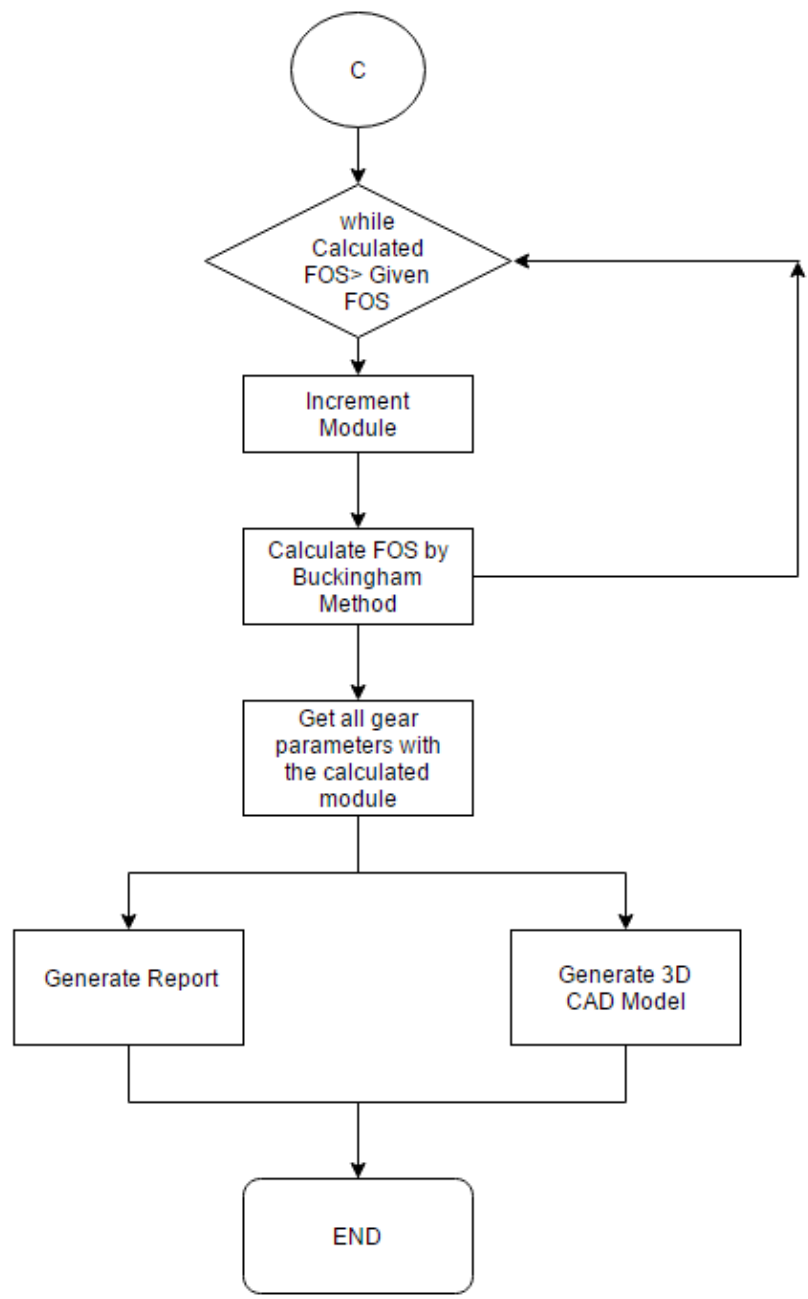

Fig. 2 Flowchart followed by the package

The program runs and calculates modules according to the above procedure. The module which is obtained finally is used to calculate the geometric parameters of the gear pair.

The module is also exported to CAD generation program which is basically a Python script which manipulates the backend code of standard CAD modeling software. The output of the CAD generation program is shown in figure.

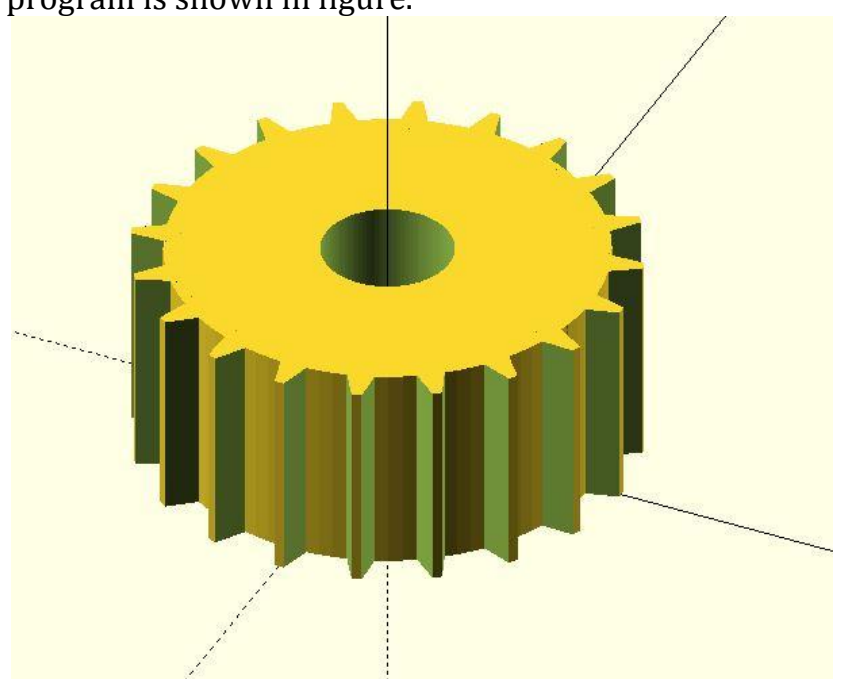

Fig. 3 Automatically generated 3D-CAD Model 


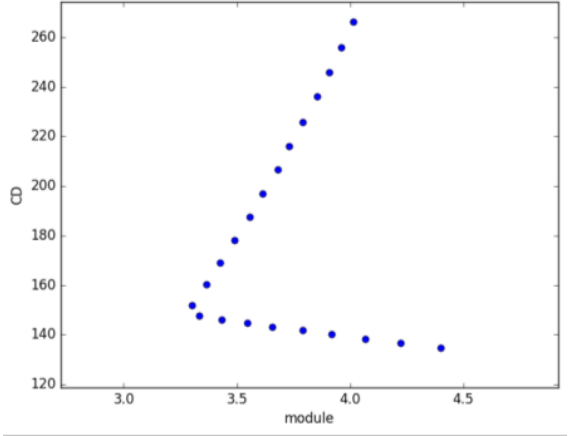

Fig4. Optimization of Center Distance (Power=10kW)

The Process involved in optimizing centre distance is by keeping Power constant with variation of no. of teeth $(\mathrm{T})$ and module $(\mathrm{m})$.The graph obtained clearly shows the optimized value of Centre Distance $(C D=152 \mathrm{~mm})$ and module $(\mathrm{m}=3.3)$ for given Power $(\mathrm{P}=10 \mathrm{~kW})$.

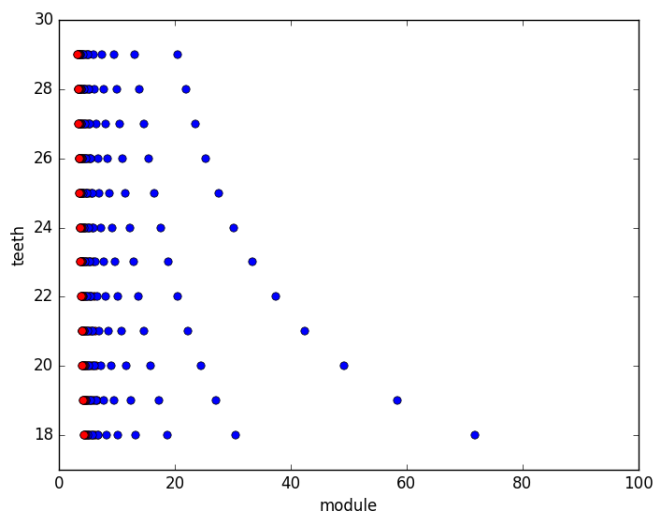

Fig 5. Variation of face width affection of basic module (By normal procedure) and Buckingham module

The graph is obtained by varying the face-width factor $(b=' x ' m)$ from $(x=5$ to $x=15)$ and the pinion teeth from( $\mathrm{t}=18$ onwards).Two values of module are obtained by variation:

1)Module by normal procedure (only velocity factor method(Red color)

2)Module by Buckingham procedure(Blue color)

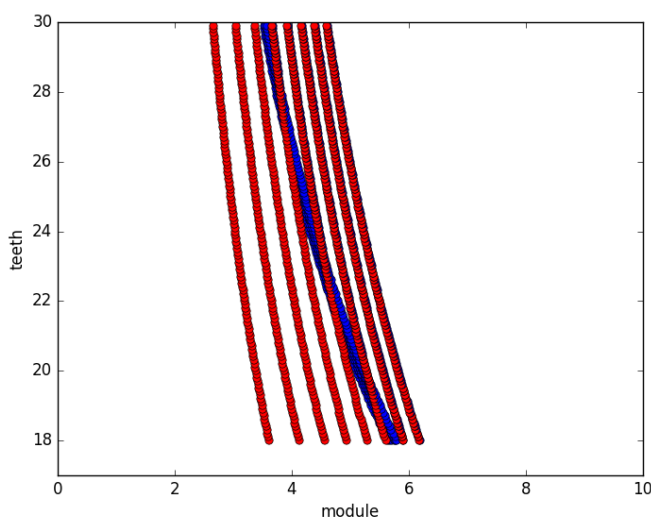

Fig 6 . Power variation from $5 \mathrm{KW}$ to $20 \mathrm{KW}$ and its effect on basic module (By normal procedure) and Buckingham module
The graph is obtained by varying the power from and the pinion teeth from(t=18 onwards).Two values of module are obtained by variation:

1)Module by normal procedure(Red color)

2)Module by Buckingham procedure(Blue color)

\section{Formulae used}

1. Bending Strength:

$$
S_{b}=m b \sigma_{b} Y
$$

2. Wear Strength:

$$
S_{w}=b Q d_{p} K
$$

3. Buckingham Dynamic Load:

$$
P_{d}=\frac{21 v\left(C e b+P_{t}\right)}{21 v+\sqrt{\left(C e b+P_{t}\right)}}
$$

where,

$\mathrm{m}=$ module

$\mathrm{b}=$ face-width

$\mathrm{Y}=$ Lewis form factor

$\mathrm{V}=$ pitch-line velocity

$\mathrm{C}=$ deformation factor

$\mathrm{e}=$ sum of errors between two meshing teeth

$\mathrm{P}_{\mathrm{t}}=$ tangential force on gear teeth

\section{Conclusions}

The authors can write the conclusion as a whole in a paragraph or by making points. An example is given as under.

1) For optimized safe design face-width factor must be taken between 12 to 15 .

2) Optimum BHN is 550 to 400 for lower number of pinion teeth to higher number of pinion teeth.

3) Optimum Ep(Young's modulus of pinion) and Eg(Young's modulus of gear) lies between 500Gpa to 100Gpa and Effect of Ep and Eg decreases with increasing number of teeth.

4) For Grade 1-5 both module with and without Buckingham load is same.

5) For Grade 6-12 module considering Buckingham Load =primary module +1

6) Effect of Buckingham Load decreases with increasing power.

7) Optimum centre distance varies with power.

\section{References}

Sushil Kumar Tiwari, Upendra Kumar Joshi (2012), Stress Analysis of Mating Involute Spur Gear teeth, International Journal of Engineering Research and Technology, Volume 1, Issue 9, Pages 1-12

Mr. Bharat Gupta, Mr. Abhishek Choubey and Mr. Gautam V. Varde (2012), Contact Stress Analysis of Spur Gear, International Journal of Engineering Research and Technology, Volume 1, Issue 4, Pages 1-7. 
Ali Raad Hassan, (2009) Contact Stress Analysis of Spur Gear Teeth Pair, World Academy of Science, Engineering and Technology, 3,104-107

Fredette.L and Brown.M.(1997), Gear Stress Reduction Using Internal Stress Relief Features, Journal of Mechanical Design, vol. 119, pp. 518-521

Christos A. Spitas and Vasilis A. Spitas(2006), Generating Interchangeable 200 Spur Gear Sets with Circular Fillets to Increase Load Carrying Capacity, International Conference on Gears, Vol. I, pp. 927-941
M.Savage , K.L. Rubadeux and H.H.Coe(1995), Bending Strength Model for Internal Spur Gear Teeth, Army Research Laboratory ,Technical Memorandum San Diego, California

Sushil Kumar Tiwari, Upendra Kumar Joshi (2012), Stress Analysis of Mating Involute Spur Gear teeth, International Journal of Engineering Research and Technology, Volume 1, Issue 9, Pages 1-12 